\title{
ACUTE COLD UNILATERAL ISOLATED PTOSIS WITH RAPID RESPONSE OF HIGH DOSE OF STEROID
}

\author{
Alsamman, A..$^{(*)} \&$ Radwan Y. \\ Ophthalmology dept., Faculty of Medicine, Sohag Univ., Sohag, Egypt \\ ${ }^{(*)}$ E-mail: alahmady20@yahoo.com
}

\begin{abstract}
A 23-year-old female presented with acute onset left painless ptosis of 5 days duration. No other ocular or neurological symptoms were associated. Ophthalmological examination was free with noticed brisky pupil reaction. Both systemic and neurological examinations were free. CT and MRI imaging were free with no swollen levator or any extra ocular muscles. After showing no improvement over 5 days with no medications the patient started oral prednisolone in low dose $15 \mathrm{mg}$ per day after one week no improvement detected then high steroid therapy $45 \mathrm{mg}$ per day was given with marked improvement within couple of days. The same dose was prescribed for 5 days with gradual withdrawal over 2 weeks. The patient remains symptomless all over six months which is the follow-up period. Conclusion: Acute ptosis may indicate serious pathology or may be simple pathology with rapid improvement.
\end{abstract}

Keywords: Ptosis, Steroids, Levator function, Diplopia

\section{Background}

Acute ptosis may occur with severe underlying pathology and a careful evaluation should be done including history,

\section{Case Presentation}

A 23-year-old female was presented at the Ophthalmology Department Sohag Faculty of Medicine at 6 December 2016 with acute onset painless left ptosis of 5 days. The ptosis occurred suddenly after waking up and was constant all over the day with no diurnal variation with no history of fatigability or variability no any other ocular symptoms like double, defective vision or abnormal eye position. Also examination and advanced investigations to exclude severe pathology and accordingly we can manage appropriately.

there is no neurological symptoms like headache or neuralgia. Prior to this complaint our patient didn't have any eye complaints and was not a contact lens wearer. On ophthalmological examination the palpebral fissure measured $7 \mathrm{~mm}$ on the right and $2 \mathrm{~mm}$ on the left. The superior margin-reflex distance was 4.5 $\mathrm{mm}$ on the right and $1 \mathrm{~mm}$ on the left. Levator function measured $13 \mathrm{~mm}$ in right eye and $5 \mathrm{~mm}$ in left eye. Visual 
acuity was 6/6 (0.1 logMAR) in each eye and pupils were rounded regular and brisky reactive to light in both direct and indirect reflexes and reactive to accommodation. Examination of ocular motility was normal with normal cover test with no deviation. Perfect single binocular vision with no diplopia. Normal intraocular pressure, normal fundus examination. An orthoptic assessment and HESS chart confirmed the above findings with full eye movements and an isolated ptosis. Cranial nerves examinations were normal. Examination of the lid except its ptosis position the lid was normal in appearance normal skin and tarsal thickness, no mechanical cause of ptosis such as mass, palpable swelling, thickening or subtarsal abnormality. The lid crease height was in normal position at $8 \mathrm{~mm}$ and symmetrical bilat-erally. Figure (1) show patient with ptosis on presentation while figure (2) after one month with complete resolution, written consent was taken from patient to publish her photos and ethics committee was taken from Sohag Faculty of Medicine.

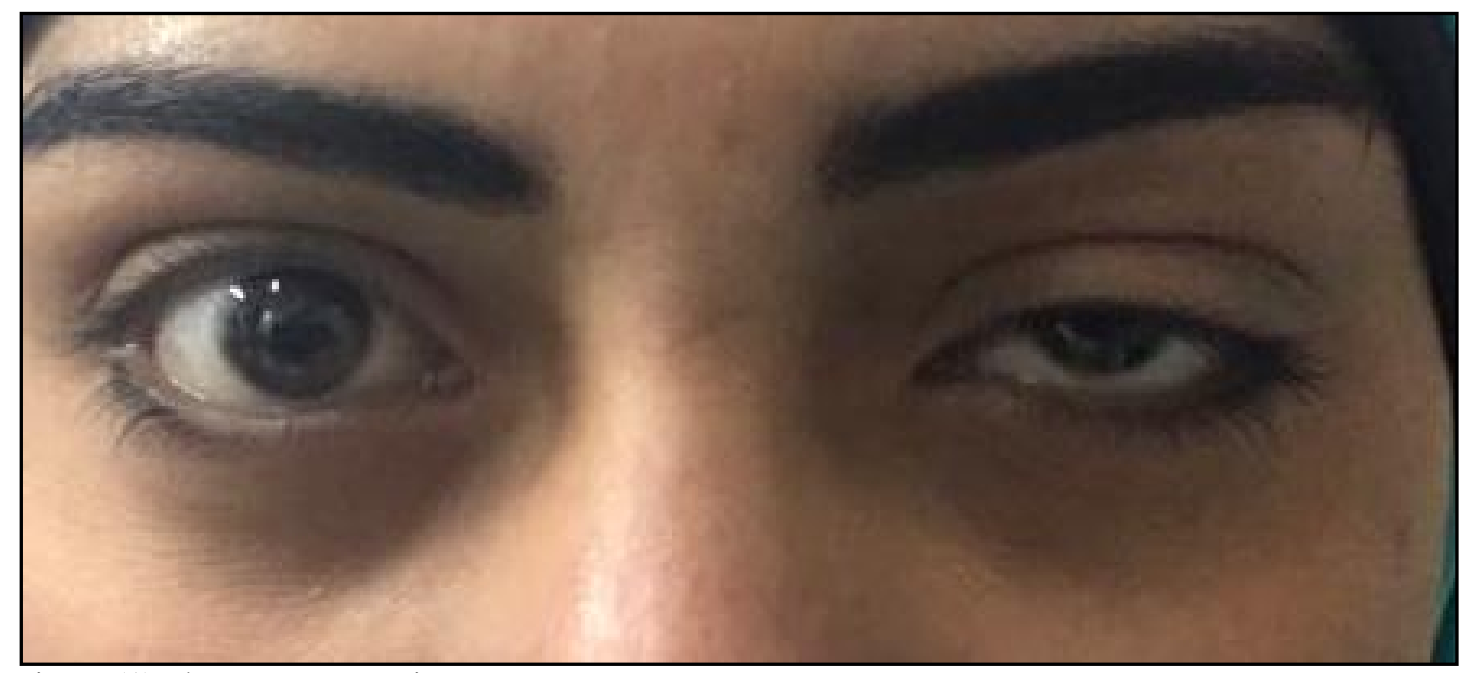

Figure (1) Photo at presentation

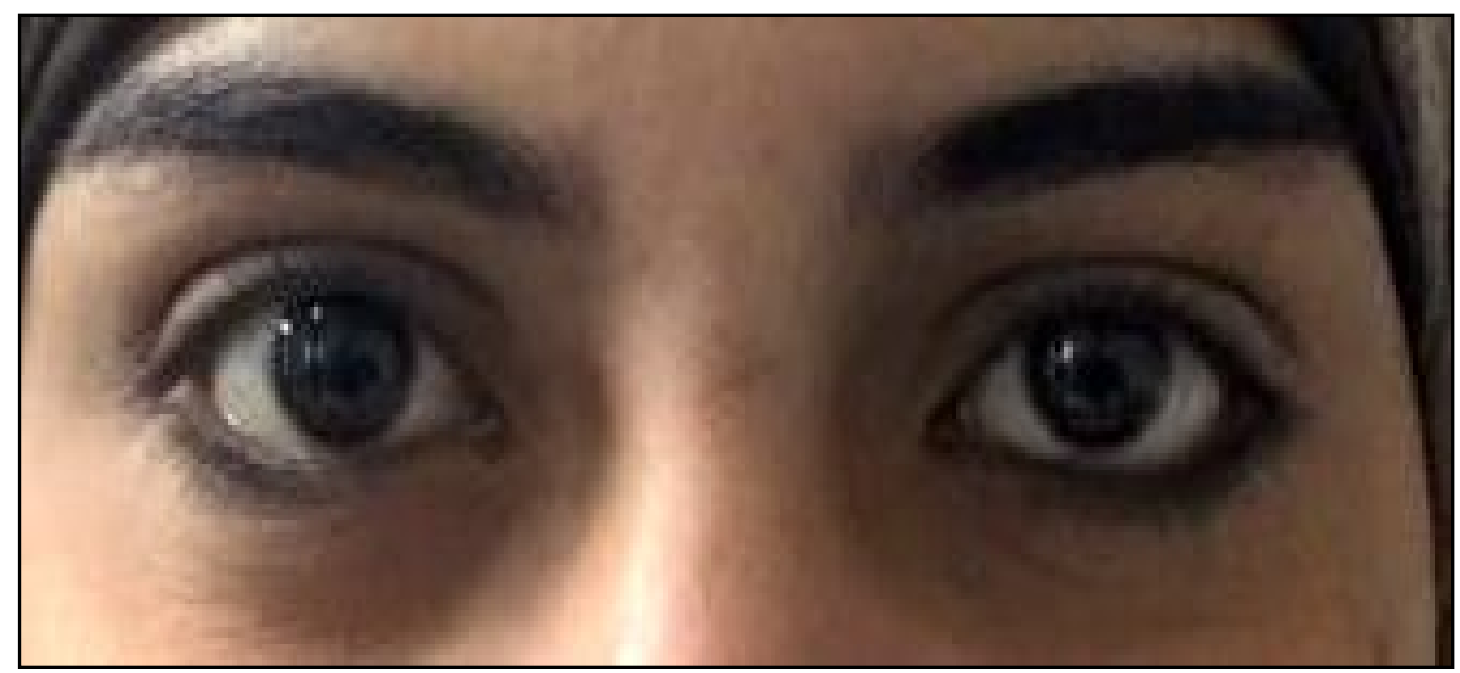

Figure (2) Photo after two weeks

\section{Investigations}

Both CT and MRI were done to rule out the possibility of vascular aneurysm eg; posterior communicating artery aneurysm and exclude any compression of the nerves along their course. This showed a normal anatomical appearance, no any abnormal mass and no aneurysm with normal cerebral vasculature. There was 
normal levator palpabrae superioris with normal architecture of all extra ocular muscles. There is no nerve compression, nor venous dilatation or other pathology. Essential Laboratory investigations were done for the patient including complete

\section{Differential diagnosis}

In Neurological ptosis in case of partial third cranial nerve palsy the paralysis usually involves three recti muscles, the superior, inferior and medial recti with diplopia and strabismus. If space occupying lesion is the cause of compression of the nerve fibres, papillary fibres located at the periphery of the third nerve will be the first affected leading to pupil dilation. The most common vascular lesion is posterior communicating artery aneurysm. In this case the pupil reaction is brisky and there was no headache which is mostly present in vascular aneurysm, but aneurysm must be considered and excluded as it is a very dangerous life threatening vascular disease and early detection is mandatory to avoid dangerous complications [1]. If medical neuropathy is the cause of third nerve affection, the commonest cause is diabetes mellitus in which microvascular abnormality is the underlying pathology also hypertension, dyslipidaemia and smoking may be a responsible cause [2,3]. Horner's syndrome is another neurological ptosis with rapid diagnosis with a constricted pupil. The diagnosis can be easily confirmed with topical apraclonidine which is a pharmacological test, resulting in rapid dilation of the narrow pupil due to denervation hypersensitivity of the dilator pupillae muscle [4]. In myasthenia gravis, ptosis and diplopia are frequently the initial presenting symptoms of both the ocular and the generalized forms of the disease. The ptosis may be unilateral or bilateral and frequently asymmetric. Unusual presentations of MG have been reported. Examples include unilateral abducens nerve palsy with diplopia and blood count total and differential, lipids, thyroid function tests, liver function tests, glucose, urea, electrolytes, $\mathrm{C}$ reactive protein and erythrocyte sedimentation rate. Autoimmune screen eg; thyroid antibodies and antinuclear antibody was normal.

ataxia. The hallmark feature of myasthenia gravis is the fluctuating weakness and fatigability of the muscles, typically worsening with activity, Theedrophonium (Tensilon) and ice pack tests are quick and easy to exclude MG [5]. This is the condition for this case. Mitochondrial myopathies commonest form is late onset bilateral progressive external ophthalmoplegia (PEO). Up to $90 \%$ of PEO patients have additional weakness of the bulbar, facial or a limb muscle which is excluded in this case. Its age of onset ranges variably. PEO is presented by ptosis and weakness of extraocular muscles affecting most of extraocular movements with relative sparing of downgaze [6]. Oculopharyngeal muscular dystrophy is combination of ptosis and pharyngeal weakness associated with weakness and wasting of face, neck, proximal, and distal limb muscles ophthalmoparesis which is evident in the autosomal dominant type Genetic testing is necessary to exclude it [7]. Orbital infection is an important differential of orbital myositis in which patients are presented with more diffuse symptoms and signs. The major symptoms are diplopia $(85 \%)$, acute to subacute orbital and/or retroorbital pain $(95 \%)$, conjunctival injections closely related to the affected eye muscle, and proptosis [8]. Recurrence can affect sporadic muscles with sporadic alternation between any of both eyes [9]. In such cases intravenous antibiotics are given and closely monitored before the diagnosis of idiopathic orbital inflammation is made. However, the patient was systemically well with absent orbital signs, swelling or pain, no erythema, and normal CBC so we did not recommend treatment with intravenous antibiotics. Given the CT scan 
findings and steady state of symptoms in the 5 days from onset to starting steroids we assumed the diagnosis of

\section{Treatment}

After showing no improvement over 5 days and further 7 days with low dose steroid (15mg prednisolon per day) and in view of the CT scan result, the patient was started on $45 \mathrm{mg}$ oral

\section{Outcome and follow-up}

The patient completely resolved from ptosis after 3 days of high dose steroid with no recurrence in the following

\section{Discussion}

Orbital myositis is considered as non-specific inflammation which may affect the orbit. It firstly may involve one of the extraocular muscles. Females more affected than males .It affects mainly young ages [10-12]. Orbital pain typically presents which increased by eye movement, the conjunctiva is usually injected and oedematous over the affected muscle, diplopia sometimes present due to defective function of the involved inflamed muscle and in severe cases mild proptosis may be present. It is usually unilateral, acute and most commonly affects the medial rectus, followed by the superior and lateral rects and rarely the obliques and inferior rectus [13]. Levator palpebrae superioris myositis may occur resulting in isolated Ptosis. The presentation of ptosis caused by levator palpabrae superioris myositis rarely described in the literature [14-18]. Rice CD et al, and Umehara $F$ et al, described levator function limitation and lid lag on downgaze in their case reports however in this case only limitation of elevator function was reported with no lid lag in down gaze $[14,15]$. Wheatcroft $\mathrm{S}$ et al, reported Lid swelling as a feature in their case which was absent in this case [16]. Yoon JH et al, described discomfort and pain was a characteristic feature of their case presentation which is absent in our case [18]. In this case the CT scan showed normal appearance of the superior rectus infection was very unlikely and did not warrant further investigation.

prednisolone reducing to $20 \mathrm{mg}$ after 1 week. The ptosis remarkably improved and steroids were slowly reduced and stopped over the following 4 weeks.

six months with no need for any type of medications.

muscle with no change in thickness or echogenicity however the action of the muscle is severely affected .We agree with Siatkowski et al [19] in their study on 75 patients with orbital myositis and reported that the function of the extraocular muscles is greatly affected during the whole course of the disease. In our case the function of the levator muscle was severely affected. We differ with Jennifer Helen Court and David Janicek's case [4] of acute unilateral isolated ptosis in which the first 10 days the extraocular muscle function was normal, followed by a paretic phase in the following days. Also CT showed thickening of superior rectus and the adjacent levator, while our patient presented with affected leavator function from the start with no abnormal findings in CT scans. Both cases improved remarkably after systemic high dose steroids; however they reported some cases of orbital myositis become refractory and may require repeated courses of steroid treatment. Sean K. Golden et al described an unusual case of Myasthenia Gravis similar to our case presentation but his case was presented with an acute onset of persistent unilateral ptosis without diurnal variation or other fluctuation in severity associated with ipsilateral facial droop [5]. Harding et al in 1984, reviewed the records of 60 patients with 
mitochondrial cytopathy at the National Hospital, Queen Square, London, and found that $54(90 \%)$ of the patients had ocular or oculomotor abnormalities and CPEO was the presenting feature in about one third of these patients. In most of the patients, ptosis is usually the first evidence of involvement and may precede ophthalmoparesis by months to years. Characteristically, the ptosis is bilateral and progresses to complete ptosis in most cases. Initial asymmetric ptosis or unilateral ptosis can occur but is less common [20].

\section{Conclusion}

An eye ptosis can be a simple isolated condition with or without any inflammatory signs of the lavatory muscle, part of localized disease, or just a starting sign for dangerous series of events. Further investigations to exclude other inflammatory, infectious, vasculitic or neoplastic, conditions are recommended for those cases.

\section{References}

1. Good, E. Ptosis as the sole manifestation of compression of the oculomotor nerve by an aneurysm of the posterior communicating artery. J Clin Neuroophthalmol 1990; 10: 59-61.

2. Jacobson, D., Broste, S. Early progression of ophthalmoplegia in patients with ischemic oculomotor nerve palsies. Arch Ophthalmol.1995; 113 (12): 15351537.

3. Patel, S., Holmes, J., Hodge, D., Burke, J. Diabetes and hypertension in isolated sixth nerve palsy: a population-based study. Ophthalmology. 2005; 112 (5): 760-763.

4. Court, J., Janicek, D. Acute unilateral isolated ptosis. BMJ Case Reports 2015; 10: 1136-2014-207720

5. Golden, S., Reiff, C., Painter, C., Repplinger, M. Myasthenia gravis presenting as persistent unilateral ptosis with facial droop. J Emerg Med. 2015; 49 (1): e23-e25.

6. Khwaja, G. Chaudhry, N., Koche, S., Singh, G., Sen, K., Mehndiratta, M., Saran, R. isolated unilateral ptosis as the initial manifestation of mitochondrial cytopathy. JIACM 2007; 8 (4): 349-351

7. Schoser, B. Ocular myositis: Diagnostic assessment, differential diagnoses, and therapy of a rare muscle disease - five new cases and review. Clin Ophthalmol. 2007; 1 (1): 37-42.

8. Weinstein, G., Dresner, S., Slamovits, T., Kennerdell, J. Acute and subacute orbital myositis. Am J Ophthalmol. 1983; 96: 214-17.

9. Moorman, C., Elston, J. Acute orbital myositis. Eye. 1995; 9: 96-101.

10. Costa, R., Dumitrascu, O., Gordon, L. Orbital myositis: Diagnosis and man- agement. Curr Allergy Asthma Rep 2009; 9: 316-323

11. Scott, I., Siatkowski, R. Idiopathic orbital myositis. Curr Opin Rheumatol 1997; 9: 504-512

12. Dubey, A., Eidsness, R., Koul, R. Idiopathic orbital myositis and review of literature. Internet J Ophthal Vis Sci 2009; 8: 1-5

13. Trokel, S., Hilal, S. Recognition and differential diagnosis of enlarged extra ocular muscles in computed tomography. Am J Ophthalmol 1979; 87: 503-512

14. Rice, C., Gray, L. Isolated levator myositis. Ophthal Plast Reconstr Surg 1988; 4: 167-170

15. Umehara, F., Tokimura, Y., Osame, M. Acute isolated levator palpebral myositis. Rinsho Shinkeigaku 1998; 38: 63-65.

16. Wheatcroft, S., Elston, J. Unilateral ptosis due to isolated involvement of the levator muscle in acute orbital myositis. Br J Ophthalmic 1999; 83: 631-632

17. Almekhlafi, M., Fletcher, W. Levator palpebrae myositis. Neurology 2008; 71 (15): 1202

18. Yoon, J., Moon, H., Chi, M. Unilateral ptosis due to isolated levator myositis. $\boldsymbol{J}$ Korean Ophth Soc 2012; 53: 707-711

19. Siatkowski, R., Capo, H., Byrne, S., Gendron, E., Flynn, J., Muñoz, M., Feuer, W. Clinical and echographic findings in idiopathic orbital myositis. Am J Ophthalmol 1994; 118: 343-350

20. Khwaja, G. Prolonged, persistent, isolated unilateral ptosis as the initial manifestation of mitochondrial cytopathy. JIACM 2007; 8 (4): 349-351 